\title{
Molecular Iodine-Catalyzed Reactions: Our Own Account
}

\section{Bimal Banik $\mathbf{K}^{1,2,3,4^{\star}}$}

${ }^{1}$ Department of Chemistry and Chemical Biology, Stevens Institute of Technology, Hoboken, New Jersey, USA

${ }^{2}$ The University of Texas MD Anderson Cancer Center, USA

${ }^{3}$ Department of Chemistry, The University of Texas-Pan American, USA

${ }^{4}$ Community Health Systems of South Texas, Edinburg, Texas 78539, USA

\author{
Abstract \\ Molecular iodine has proven to be extremely useful in our research for the synthesis of diverse organic compounds \\ of significant importance. The acidity of molecular iodine is responsible for the success of these reactions.
}

Keywords: Molecular iodine; Catalysis; Carbohydrate; Synthesis; Beta lactam; Alkaloids; Microwave

\section{Introduction}

Molecular iodine is highly economical and readily available in almost all laboratories in the world. Our research group has been conducting molecular iodine-mediated useful reactions for the past many years. Synthesis of diverse organic molecules in chiral and achiral form is realized by iodine-catalyzed reaction very effectively. Moreover, iodine-mediated reactions can be easily performed under microwave irradiation method making this process is attractive. Illustrative examples of reactions from our laboratory only are provided that include various compounds of interests.

\section{Results and Discussion}

Molecular iodine is a very versatile catalyst and readily available with extremely low cost. It has been possible to conduct numerous successful reactions with molecular iodine-catalyzed reactions for the preparation of diverse organic compounds. Molecular iodine can produce acid in a reaction medium. It has also coordinating properties. Some examples are given below without describing the details of these reactions.

\section{Protection and deprotection of carbonyl compounds}

Protection and deprotection of the aldehydes and ketones are important chemical methods in synthetic organic chemistry. Mild acidic reagents were found to catalyze these processes effectively. Based upon the importance, numerous methods were developed for this purpose. In general, protection of aldehydes and ketones were performed as acetal, ketals, thioketals, and mixed ketals. Molecular iodine was found to catalyze these processes very well [1]. In general, it was found that acetal formation with aldehydes proceeds much faster. Ketal, thioketal and mixed ketal formation within an identical series of compounds followed at the same rate. In general methanol, ethanol, ethylene glycol, ethylene thiol, ethylene thioalcohol were used as the other starting compounds in anhydrous tetrahydrofuran, dichloromethane, dioxane, and dimethylsulfoxide. The reaction with ketones was slower than aldehydes within a similar group of compounds. Sterically unhindered ketones reacted faster than sterically hindered ketones and produced acetals and ketals more rapidly. These differences of reactivity among aldehydes and ketones were used to induce intramolecular and intermolecular selectivity among many compounds. These selectivities were used for the preparation of many important structures. Deprotection of the acetal, ketal, thioketal and mixed ketals were performed with molecular iodine [2]. Compounds with these types of protective groups yield the original carbonyl compounds in the presence of iodine. This was possible because protection and deprotection of aldehydes and ketones are reversible reactions. Mechanistically, these reactions involve a nucleophilic addition of an acidic reagent to the carbonyl group or the protective groups. It is believed that the reactions can proceed with iodine through a Lewis acid type of reactions or Bronsted acid-mediated reactions. Our research in this area culminated in a few methods of molecular iodine-catalyzed protection and deprotection reactions. Protection and deprotection reactions with molecular iodine were used in beta-lactam chemistry. These methods were necessary for preparation of amino sugars, amino acids, alkaloids and polycyclic beta-lactams [3].

\section{Glycosylation reactions}

Stereospecific synthesis of oxygen glycosides was performed with hydroxy beta lactams and diverse glycals using molecular iodine as the catalyst. Racemic 3-hydroxy beta lactams on reaction with glycal in the presence of catalytic amounts of iodine produced two diastereomeric separable O-glycosides [4]. The O-glycosides were cleaved by mild acid to the corresponding hydroxy beta-lactams in both enantiomeric forms. This process was successfully applied for the synthesis of all enantiomeric forms that are possible for a 3, 4-disubstituted beta lactam. Optically active beta lactams that are present in Taxol and Thienamycin side chains were made using this method. The role of iodine in these chemical transformations was not established. But, it was important to note that the reactions as mentioned above did not proceed in the absence of catalyst. In some instances, iodometric titration was performed to identify whether iodine was really consumed in the reaction. Surprisingly, sodium thiosulfate titration method indicated no iodine was consumed, yet the reaction proceeded completely.

\section{Condensed heterocycles}

Molecular iodine was successful in catalyzing the reaction between isatin and hydoxyproline derivatives to form pyrrole-fused oxoindoles in high yield [5]. It was believed that the amino acid group of proline reacted with the keto group of isatin and formed a cyclic intermediate. This unstable intermediate then finally produced the product through the activation exerted by iodine. It was remarkable that the iodinecatalyzed reaction between 3 -keto-beta lactams on reaction with hydroxy proline produced 3-pyrrole substituted beta lactams [6].

*Corresponding author: Bimal Banik K, Community Health Systems of South Texas, Edinburg, Texas 78539, USA, Tel: 19566274094; E-mail: bimalbanik10@gmail.com (or)bimal.banik@chsst.org

Received July 25, 2016; Accepted August 01, 2016; Published August 04, 2017

Citation: Bimal Banik K (2017) Molecular lodine-Catalyzed Reactions: Our Own Account. Mod Chem appl 5: 227. doi: 10.4172/2329-6798.1000227

Copyright: $\odot 2017$ Bimal Banik K. This is an open-access article distributed under the terms of the Creative Commons Attribution License, which permits unrestricted use, distribution, and reproduction in any medium, provided the original author and source are credited. 


\section{Samarium-induced reactions}

Samarium-induced chemistry in the presence of iodine was useful to study reduction of carbonyl compounds and imines and reductive dimerization of carbonyl compounds and imines. These reactions did not proceed in the absence of iodine [7]. The success of these reactions was not due to the formation of bivalent samarium species.

\section{Pyrrole synthesis}

A reaction of primary amine with hexane 2,4-dione in the presence of molecular iodine produced $\mathrm{N}$-substituted pyrroles. A variety of primary amines were used to generalize the process [8].

\section{Alkaloid synthesis}

Molecular iodine was able to catalyze Michael reaction between indoles and 2,3-unsatuated ketones in excellent yield in the absence of any solvents [9]. An extremely rapid and convenient microwaveassisted method for the synthesis of indoloquinolines using molecular iodine as a catalyst in one-pot was developed [10]. The mixture of indole-3-carboxyaldehyde and two equivalents of aniline in presence of $10 \mathrm{~mol} \%$ of iodine produced indoloquinolines. An effective iodinecatalyzed method for the synthesis of quinoxalines by the condensation of 1,2-diamines with 1,2-dicarbonyl compounds was developed [11].

\section{Optically active polyaromatic alcohol}

Racemic dibenzofluorenol on reaction with a glycal in the presence of catalytic amounts of iodine afforded two diastereomers. These were separated and cleaved to the optical isomers of dibenzofluorenol [12]. Our laboratory had demonstrated synthesis and studied biological activity of polyaromatic compounds for the past many years [12].

\section{Microwave-induced method}

The reactions described above were conducted under microwave irradiation method [13]. It was crucial to observe that all these reactions were successfully performed in domestic or automated microwave oven. Clearly the microwave-induced iodine-catalyzed method was very fast, economical and produced products with better purities.

\section{Conclusion}

We demonstrated iodine-catalyzed reactions for the synthesis of diverse compounds. Most of the iodine-catalyzed reactions as described above produced products with high yields. Isolation of products from the reaction mixture was very convenient. Some reactions proceeded in the absence of any solvent with $1 \mathrm{~mol} \%$ iodine. Considering the budgetary restrictions, our iodine-catalyzed reactions are the perfect examples to cut down the cost of research maintaining the standard of good publications. In addition, it was also found that iodine-catalyzed reactions can tolerate microwave-irradiation. On this basis, some of the iodine-catalyzed reactions under microwave irradiation were completed within minutes instead of hours/days. A few compounds prepared by iodine-catalyzed reactions demonstrated anticancer and antibacterial activities.

\section{Acknowledgements}

BKB is highly grateful to numerous students and scientists who have participated in this research. I am also grateful to $\mathrm{NIH}, \mathrm{NCl}$, Kleberg Foundation, Stevens Institute of Technology, University of Texas MD Anderson Cancer Center and University of Texas-Pan American for their support to our research.

\section{References}

1. Samajdar S, Basu MK, Becker FF, Banik BK (2001) A New Molecular lodineCatalyzed Thioketalization of Carbonyl Compounds: Selectivity and Scope. Tetrahedron Lett 42: 4425-4428.

2. Banik BK, Garza R (2007) lodine-Catalyzed Facile Deprotection of Acetal and Ketal in Acetone. Chem Edu 12: 75-76.

3. Bose AK, Banik BK, Mathur C, Wagle DR (2000) Polyhydroxy Amino Acid derivatives via $\beta$-Lactams Using Enantiospecific Approaches and Microwave Techniques. Tetrahedron 56: 5603-5619.

4. Banik BK, Manhas MK, Bose AK (1997) EnantiopureHydroxy $\beta$-Lactams via Glycosylation. Tetrahedron Lett 38: 5077-5080.

5. Banik BK, Garcia I, Moarles F, Aguilar C (2007) Novel Synthesis of Substituted Pyrroles Bound to Indolinone via Molecular lodine-Catalyzed Reaction. Heterocyclic Communications 13: 109-112.

6. Bandyopadhyay D, Cruz J, Yadav RN, Banik BK (2012) An Expeditious lodineCatalyzed Synthesis of 3-Pyrrole Substituted 2-Azetidinones. Molecules 17 11570-11584.

7. Banik BK, Samajdar S, Ghatak A (2001) A Convenient Samarium-Mediated Reduction of Ferrocenyl Imines. Heterocycles 55: 1957-1961.

8. Banik BK, Samajdar S, Banik I (2004) Simple Synthesis of Substituted Pyrroles. J Org Chem 69: 213-216

9. Banik BK, Farnandez M, Alvarez C (2005) lodine-Catalyzed Michael Reaction of Indoles Under Solventless Conditions. Tetrahedron Lett 46: 2479-2482.

10. Parvatkar P, Prameswaran PS, Bandyopadhyay D, Mukherjee S (2017) lodineCatalyzed Novel Synthesis of Carbazoles. Current Microwave Chemistry.

11. Bandyopadhyay D, Mukherjee S, Rodriguez R, Banik BK (2010) An Effective Microwave-Induced lodine-Catalyzed Method for the Synthesis of Quinoxalines via Condensation of 1,2-Diamines with 1,2-Dicarbonyl Compounds. Molecules 15: 4207-4212.

12. Banik BK, Mukhopadhya C, Logan C (2007) Optical Resolution of Dibenzofluorenol: Intermediates for Anticancer Agents. Synthetic Communications 37: 3895-3900.

13. Banik BK, Barakat KJ, Wagle DR, Manhas MS (1999) Microwave-Assisted Rapid and Simplified Hydrogenation. J Org Chem 64: 5746-5753. 